\title{
ESTRUCTURA Y DINÁMICA POBLACIONAL DE LOS CAZADORES-RECOLECTORES DEL SITIO MÉDANO PETROQUÍMICA (LA PAMPA, ARGENTINA): INFERENCIAS A PARTIR DEL USO DE TABLAS DE VIDA Y OTROS INDICADORES PALEODEMOGRÁFICOS
}

\author{
POPULATION STRUCTURE AND DYNAMIC OF HUNTER-GATHERERS FROM \\ THE MÉDANO PETROQUÍMICA SITE (LA PAMPA, ARGENTINA): INFERENCES \\ FROM LIFE TABLES AND OTHER PALEODEMOGRAPHIC INDICATORS
}

\author{
Lila Bernardi ${ }^{1,2}$, Mario A. Arrieta ${ }^{1,2}$ y Melina C. Bottini ${ }^{1,2}$
}

\begin{abstract}
La paleodemografía constituye una línea de investigación muchas veces subestimada en los estudios bioarqueológicos debido a que generalmente los sitios prehistóricos carecen de registros escritos que proporcionen información precisa de las sociedades que los generaron. Dado el importante número de individuos inhumados en el sitio Médano Petroquímica ( $\mathrm{n}=120)$ y el breve periodo de ocupación del mismo (entre 363 y 419 años AP), la utilización de herramientas como las tablas de vida y de distintos índices paleodemográficos nos permitirá aportar información relevante de la dinámica poblacional para el área de estudio durante el Holoceno Tardío. Las tasas de mortalidad infantil y de fecundidad femenina, junto a otros indicadores, sugieren que se trataría de una población con tendencia hacia el crecimiento. Además, el análisis e interpretación de los resultados obtenidos apoyaría el cuadro propuesto por algunos investigadores, en el cual las sociedades de la región habrían experimentado una disminución de la movilidad residencial, el aumento de la densidad poblacional y la aparición de áreas formales de entierro durante el periodo de contacto hispano-indígena inicial.
\end{abstract}

Palabras claves: paleodemografía, cazadores-recolectores, Holoceno Tardío.

Paleodemography is a line of research that is often underestimated in bioarchaeological studies because prehistoric sites generally lack written records that provide accurate information about the societies that generated them. Given the high number of individuals buried at the Médano Petroquímica site and the short period of occupation thereof, the use of life tables and different paleodemographic indices would allow us to provide relevant information about population dynamics for the study area during the late Holocene. Infant mortality, female fertility rates, and other indicators suggest it would be a population with a tendency towards growth. In addition, the results would support the model proposed by some researchers of decreasing residential mobility in the region, increasing population density, and the appearance of formal burial areas during the initial period of Spanishindigenous contact.

Key words: Paleodemography, hunter-gatherers, Late Holocene.

La paleodemografía es la disciplina dedicada al estudio de las dinámicas poblacionales de sociedades humanas del pasado (Frankenberg y Konigsberg 2006). Este subcampo de la bioantropología surgió del creciente interés de los investigadores por el estudio sistemático y global de las sociedades antiguas para una mejor comprensión del desarrollo tanto biológico como cultural. Los estudios paleodemográficos revisten una gran importancia bioarqueológica, ya que proporcionan las herramientas para inferir propiedades de las sociedades prehistóricas tales como estructura social y composición biológica, variaciones espaciotemporales, estados de salud, esperanza de vida, tasas de mortalidad y fecundidad, como también la influencia de los cambios culturales, socioeconómicos y ambientales sobre la estructura poblacional (Angel 1969; Buikstra y Konigsberg 1985; Frankenberg y Konigsberg 2006; Hoppa 2002; Meindl y Russell 1998; Milner et al. 1989). Dado que los datos que utiliza la paleodemografía se obtienen mayormente del análisis de restos óseos provenientes de contextos arqueológicos (Hoppa 2002), donde generalmente

\footnotetext{
$1 \quad$ Laboratorio de Bioarqueología, Facultad de Ciencias Exactas, Físico-Químicas y Naturales, Universidad Nacional de Río Cuarto,Argentina.1bernardi@exa.unrc.edu.ar; marrieta@exa.unrc.edu.ar; meli_bottini@hotmail.com

2 CONICET - Consejo Nacional de Investigaciones Científicas y Técnicas. Buenos Aires, Argentina.
}

Recibido: octubre 2019. Aceptado: septiembre 2020. 
no hay registros escritos, usualmente estos estudios presentan muchas limitaciones. Sin embargo, varios de estos inconvenientes han sido subsanados, en menor o mayor medida, a partir de la utilización de distintos supuestos, los cuales resultan necesarios para realizar interpretaciones a nivel poblacional (i.e. uniformitarismo biológico, población estable y/o estacionaria, representatividad de la muestra, entre otros), la aplicación de modelos biomatemáticos y la mejora en las técnicas de excavación y de estimación de edad y sexo. Gracias a esto, un gran número de bioarqueólogos comenzaron a interiorizarse en el uso y la aplicación de las técnicas y parámetros que son propios de la demografía (Moore et al. 1975), a fin de poder aplicarlos al estudio de las sociedades prehistóricas.

Los estudios paleodemográficos también utilizan herramientas procedentes de otras disciplinas, tales como las tablas de vida, ampliamente utilizadas en ecología, y los estudios etnográficos (Milner et al. 1989). Todo esto ha llevado a la paleodemografía a convertirse en una disciplina que recibe y necesita del constante aporte de otras áreas del conocimiento, tales como la osteología humana, antropología cultural, etnografía, medicina, demografía, estadística, entre otras (Hernández Espinoza 1999; Rodríguez Cuenca 1994). En lo que respecta específicamente a las tablas de vida, se utilizan con frecuencia para examinar los esquemas de mortalidad, supervivencia y esperanza de vida de una población (Begon et al. 1995). Puede considerarse, además, como un instrumento para conocer la estructura interna de una población, ya que permite conocer parámetros relevantes para comprender su variación en el tiempo (Martella et al. 2012). Las distintas columnas que componen las tablas de vida, estan interrelacionadas, y algunos parámetros son utilizados para calcular otros. Dado que el análisis paleodemográfico se basa en las estimaciones de edad y sexo de los esqueletos procedentes de un sitio arqueológico, determinando la cantidad de individuos por rango etario, las tablas de vida podrían ser definidas como una herramienta matemática utilizada para representar la mortalidad de una población, analizando los efectos de la probabilidad de muerte para cada categoría etaria respecto de la supervivencia (Chamberlain 2006).

\section{Área de Estudio y Sitio Médano Petroquímica}

El sitio Médano Petroquímica es un área inhumatoria localizada en el suroeste de la Provincia de La Pampa (Argentina), a $2 \mathrm{~km}$ del límite con la Provincia de Río Negro y relativamente próximo al norte de la Provincia de Neuquén y sudeste de la Provincia de Mendoza (Figura 1a). Los restos óseos se hallaron en un médano que se elevaba aproximadamente unos $3 \mathrm{~m}$ sobre el nivel del terreno, a una altitud entre 340 y $360 \mathrm{msm}$. Luego de tres campañas $(2006,2007$ y 2009), se excavaron completamente cinco fosas con entierros secundarios múltiples de diferentes dimensiones y un entierro primario simple, recuperando un total de aproximadamente 120 esqueletos. Seis fechados radiocarbónicos indican que este sitio fue utilizado desde $419 \pm 46$ AP hasta $363 \pm 45$ AP (Bottini et al. 2020; Mendonça et al. 2010). Las inclusiones arqueológicas son escasas, limitándose a un aro de cobre, un fragmento de cuenta veneciana, puntas de proyectil líticas (tanto sueltas en la matriz sedimentaria como incrustadas en distintas piezas óseas) y una gran cantidad de cuentas de collar elaboradas sobre valva de molusco. Con respecto al tratamiento funerario, estudios tafonómicos realizados por Bottini et al. (2020) sugieren una práctica basada en la desarticulación (inferido a partir de las marcas de corte) y armado de paquetes donde los huesos largos eran acomodados en haces paralelos con los elementos óseos de la cintura pélvica y cráneos dispuestos en los extremos (Figura 1b). La inclusión general de los individuos parece ser más una consecuencia del agregado de los distintos envoltorios que una disposición ordenada de los mismos. Con respecto al estado de integridad y preservación de los restos óseos recuperados, podemos decir que si bien algunos elementos como el cráneo y la pelvis (fundamentales para las estimaciones de edad y sexo) presentaron una alta fragmentación, la misma no influyó negativamente a la hora de realizar las estimaciones sexo-etarias tan importantes para el análisis que aquí se presenta (Bernardi 2019). El análisis paleodemográfico realizado hasta el momento para el sitio muestra una distribución de edad a la muerte de tipo atricional con dos picos correspondientes a los individuos menores a un año y adultos jóvenes (Bernardi et al. 2016).

Por otro lado, el análisis de indicadores de nutrición y salubridad sugiere que los individuos de esta población padecieron situaciones de estrés metabólico de severidad moderada a alta, la cual presentaría un pico de incidencia en el momento del destete y en los años subsiguientes (Bernardi 2018). Finalmente, estudios paleodietarios a través del análisis isotópico sugieren que este grupo habría presentado una dieta relativamente homogénea basada en el consumo 

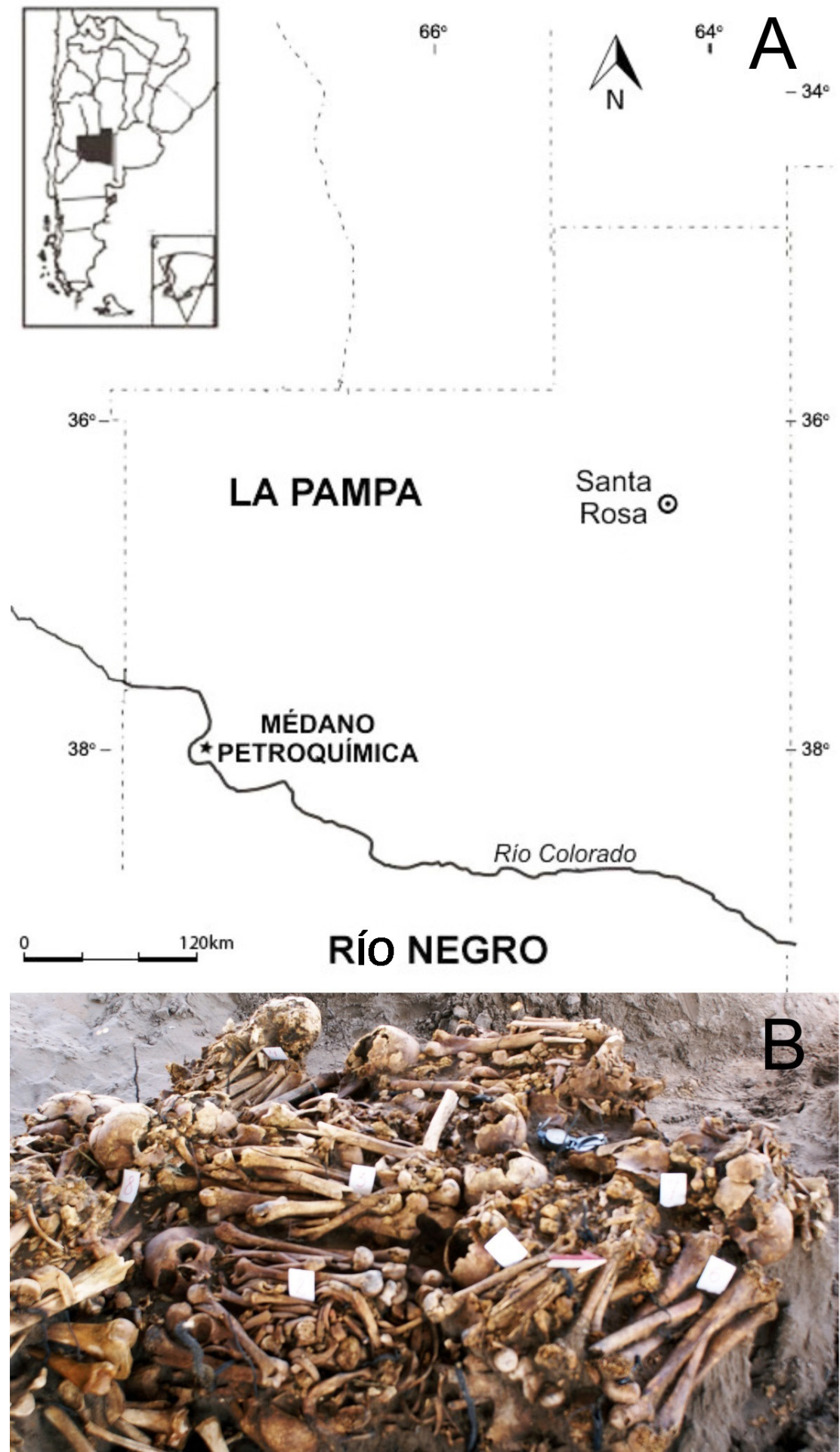

Figura 1. (A) Ubicación del sitio Médano Petroquímica, (B) vista del entierro H5 donde pueden apreciarse la concentración y disposición de los huesos.

(A) Site location Médano Petroquímica, (B) view of H5 burial where the concentration and arrangement of bones can be observed. 
generalizado de recursos terrestres, principalmente de herbívoros tales como Lama guanicoe, Rhea spp. y sus huevos, junto con animales de niveles tróficos más altos (Lynch Ianniello et al. 2019).

En lo que respecta a antecedentes regionales, varios autores proponen cambios en la organización de los grupos humanos a partir del Holoceno Tardío, entre los cuales se mencionan un aumento demográfico, reducción de la movilidad residencial y cambios en la organización sociopolítica y económica (Berón y Politis 1997; Cúneo et al. 2015; Madrid y Barrientos 2000; Martínez 2006; Mazzanti y Quintana 2001). Estos cambios podrían estar respondiendo a factores climáticos, ambientales y ecológicos (Barrientos y Pérez 2004) y podrían reflejarse en las redes de intercambio y circulación de bienes propuestas para finales del Holoceno Tardío entre Norpatagonia, el centro-sur de Chile, cuyo, la región pampeana y la costa atlántica (Barrientos 2001; Barrientos y Pérez 2004; Cúneo et al. 2015). Este sistema de intercambio y movilidad de bienes podría estar relacionado con el hallazgo de una cuenta de vidrio veneciana en Médano Petroquímica, lo cual, sumado a los fechados radiocarbónicos, sitúan al sitio en momentos de contacto hispano-indígena inicial. Este escenario nos permite considerar la presencia de expedicionarios procedentes del Viejo Mundo como otro factor de gran relevancia para los cambios en la estructura y dinámica de estas sociedades. Debido a que estos momentos representan un punto de inflexión en lo que será el destino de las sociedades originarias de la región, resulta de enorme interés poder reconocer el impacto de estos cambios mediante el abordaje bioarqueológico, en este caso específicamente a través del análisis paleodemográfico.

Si bien los cambios que se evidencian a partir del Holoceno Tardío en las sociedades de PampaPatagonia fueron estudiados desde distintas líneas de la bioarqueología, los estudios paleodemográficos constituyen un conjunto de herramientas con gran potencial, ya que pueden aportar datos a partir de los cuales se pueden realizar inferencias respecto a los cambios estructurales y a las dinámicas poblacionales para la región.

En lo que respecta al análisis paleodemográfico en nuestro país, y más precisamente en nuestra área de estudio para este periodo, los trabajos basados en la construcción de perfiles de mortalidad son abundantes. Entre ellos podemos mencionar las publicaciones de Flensborg et al. (2015), Flensborg y Bayala (2017), García Guraieb (2010), García Guraieb et al. (2015), Luna (2008, 2012), Novellino et al. (2013), Peralta
2019 y Suby et al. (2017). Por otro lado, los abordajes que utilizan la distribución sexo-etaria en sitios arqueológicos para la construcción de tablas de vida son mucho más escasos, probablemente debido a las fuertes críticas que ha tenido, desde sus comienzos, la aplicación de dichas tablas en paleodemografía. En este caso solamente podemos citar los trabajos de Gordón et al. (2019), donde se recurre a la utilización de una tabla de vida abreviada, junto a los índices JAR (juvenil/adult ratio) (Jackes 1986, 1992) y MCM (Mean Childhood Mortality) (Jackes 1986, 2011), para el análisis paleodemográfico del sitio Aquihuecó (Neuquén) y de Bernal et al. (2004) y García Guraieb (2010) para el estudio de los entierros de la cuenca del Lago Salitroso, al sur de Patagonia argentina.

Más allá de las críticas y los escasos antecedentes en el uso de tablas de vida en los estudios bioarqueológicos de la región, la utilización de este tipo de herramientas paleodemográficas para nuestro sitio de estudio representa un gran potencial, ya que Médano Petroquímica se ubica en la zona de transición de las regiones Pampa y Patagonia en un momento para el cual, como se mencionó anteriormente, se plantean importantes cambios socioculturales. Además, los fechados radiocarbónicos obtenidos (Bottini et al. 2020) sugieren un periodo acotado para el uso de este cementerio (entre los años 363 y 419 AP), lo cual fortalecería las interpretaciones y/o inferencias poblacionales que pudieran realizarse. Debido a esto, en este trabajo se plantea la utilización de tablas de vida y otros parámetros derivados de ellas para realizar inferencias sobre la estructura y dinámica poblacional de la/s sociedad/es que originaron el sitio Médano Petroquímica durante el Holoceno Tardío en momentos de contacto hispano-indígena inicial.

\section{Material y Métodos}

Se analizaron restos óseos humanos provenientes de cinco entierros (EA-1, H4, H6-1, H6-3 y H7) del sitio Médano Petroquímica, el cual habría sido generado por sociedades cazadoras-recolectoras de la región Pampa-Patagonia. Los restos óseos hallados en el entierro $\mathrm{H} 5$ no fueron incluidos en este análisis.

Para la determinación del sexo en esqueletos adultos se siguieron los criterios propuestos por Buikstra y Ubelaker (1994). Se tuvieron en cuenta la morfología de la pelvis y los distintos indicadores presentes en el cráneo y la mandíbula (Acsádi y Nemeskéri 1970; Buikstra y Mielke 1985; Buikstra y Ubelaker 1994; Loth y Henneberg 1996, 2001; 
Milner 1992; Phenice 1969; Sutherland y Suchey 1991; Walker 2005), así como los diámetros verticales de las cabezas femorales y humerales (Buikstra y Ubelaker 1994). Para esqueletos de inmaduros, la estimación del sexo se llevó a cabo aplicando las metodologías propuestas por Loth y Henneberg (2001), Luna (2008), Molleson et al. (1998) y Schutkowski (1993), las cuales consideran caracteres morfológicos de la mandíbula y del ilion.

Para la estimación de la edad a la muerte se utilizaron la secuencia de erupción y formación dental (Ubelaker 1999), el estado de desgaste dental (Lovejoy 1985; Mays 2002) y caracteres del os coxae, tales como indicadores de la superficie auricular (Buckberry y Chamberlain 2002; Lovejoy et al. 1985; Meindl y Lovejoy 1989) y de la sínfisis púbica (Brooks y Suchey 1990; Gilbert y McKern 1973; McKern y Stewart 1957; Todd 1921 a, 1921b). Para el resto de las unidades anatómicas se utilizaron los criterios propuestos por Johnston (1962) y Krogman e Isçan (1986), basados en el estado de fusión de las epífisis para distintos elementos óseos humanos. Para la estimación de la edad en inmaduros se utilizaron los métodos propuestos por Fazekas y Kósa (1978) y Scheuer y Black (2000), los cuales se basan en el examen y determinación del tamaño óseo. Estos resultados, junto con las notas de campo, fueron utilizados para llevar a cabo el ensamblaje bilateral e intermembral (Todd y Frison 1992; Osterholtz 2019) y posteriormente realizar la estimación del número mínimo de individuos presentes en la muestra estudiada.

Para organizar los datos en las tablas de vida se utilizarán los siguientes intervalos de edad: 0-0,9 años,
1 a 4,9 años, de 5 a 9,9 años, de 10 a 14,9 años, de 15 a 19,9 años, de 20 a 34,9 años (adultos jóvenes), de 35 a 49,9 años (adultos medios) y, por último, más de 50 años (adultos mayores). La variación en la cantidad de años que comprende cada rango etario responde principalmente a las diferencias en la precisión de las distintas metodologías para estimar la edad en individuos adultos e inmaduros. Por tal motivo, para adultos se utiliza el criterio de clasificación utilizado por Buikstra y Ubelaker (1994) (adultos jóvenes, medios y mayores) y categorías quinquenales para los inmaduros (excepto en la primera categoría que abarca desde perinatos hasta el primer año de vida). Dentro de la categoría de 0 a 0,9 años también se diferenciará, en la discusión, a los perinatos, los cuales están representados por aquellos individuos cuya edad al momento de la muerte estimada fue de 36-38 semanas de gestación hasta tres meses. La distinción de estas categorías se debe a que los infantes menores a un año son generalmente más susceptibles a cambios ambientales, enfermedades, restricciones nutricionales, entre otros (FitzGerald et al. 2006; Sánchez Romero 2006), lo cual ha llevado a postular que la representatividad de la muestra dependerá también del porcentaje de individuos menores de un año que está presente (Volk y Atkinson 2013).

Para construir las tablas de vida se calculó, teniendo en cuenta cada rango etario (x), el número de defunciones (Dx), el porcentaje de defunciones (dx), el porcentaje de supervivientes (lx), la probabilidad de fallecimiento (qx) y la esperanza de vida $\left(\mathrm{e}^{\mathrm{o}}{ }_{\mathrm{x}}\right.$. Estos parámetros, junto con la tasa de crecimiento poblacional (Tc), se detallan en la Tabla 1.

Tabla 1. Descripción de los parámetros paleodemográficos utilizados, tomados de Ubelaker (2007). Description of the paleodemographic parameters used, taken from Ubelaker (2007).

\begin{tabular}{|c|c|}
\hline Parámetro paleodemográfico & Cálculo según Ubelaker (2007) \\
\hline Dx & Número de esqueletos dentro del rango $\mathrm{x}$ \\
\hline $\mathrm{dx}$ & Porcentaje de esqueletos en cada rango de edad \\
\hline $1 \mathrm{x}$ & Porcentaje de individuos vivos en cada intervalo de edad $\left(1_{x}=1_{x-1}-d_{x-1}\right)$ \\
\hline $\mathrm{qx}$ & Probabilidad de muerte en cada rango $(\mathrm{dx} / \mathrm{lx})$ \\
\hline Lx & Cantidad de años vividos por los individuos de cada rango $\left(\mathrm{L}_{\mathrm{x}}=\mathrm{c} *\left(1_{\mathrm{x}}+1_{\mathrm{x}+1}\right) / 2\right)^{1}$ \\
\hline Tx & Número de años que restan por vivir por intervalo $\left(T_{x}=\Sigma L_{x}\right)$ \\
\hline $\mathrm{e}_{\mathrm{x}}^{\mathrm{o}}$ & $\begin{array}{l}\text { Número de años que los individuos de cierto rango de edad pueden esperar vivir } \\
\left(\mathrm{e}_{\mathrm{x}}^{\mathrm{o}}=\mathrm{Tx} / \mathrm{lx}\right)\end{array}$ \\
\hline M & $\begin{array}{l}\text { Tasa bruta de mortalidad: promedio de individuos que mueren de cada mil, en cada año(M= } \\
1000 / \mathrm{e}^{\mathrm{o}} \text { ) }\end{array}$ \\
\hline $\mathrm{T}_{\mathrm{c}}$ & $\begin{array}{l}\mathrm{T}_{\mathrm{c}}=1,484 \mathrm{x}+1,485 \pm 0,0006 \\
\left(\mathrm{x}=\log \left[200 *\left(\mathrm{D}_{5-14} / \mathrm{D}_{20-\mathrm{w}}\right) *\left(\mathrm{D}_{60-\mathrm{w}} / \mathrm{D}_{20-\mathrm{w}}\right)\right]^{0,03}\right)\end{array}$ \\
\hline
\end{tabular}


Además, Bocquet-Appel y Masset (1977) propusieron una serie de estimadores de interés paleodemográfico, los cuales se basan en el llamado Índice de Juventud (IJ), y son descritos en la Tabla 2.

Como puede observarse en la fórmula para estimar el IJ, este índice se calcula dividiendo el grupo de individuos cuya edad fue estimada en el rango comprendido entre cinco y 14 años por el grupo mayor o igual a 20 años correspondiente a los adultos de la muestra analizada. En este índice no se tiene en cuenta al grupo de individuos cuyo rango de edad va de 0 a 4,9 años, porque de este modo los autores pretendían evitar incluir en los cálculos de los distintos estimadores al grupo cuyos restos óseos son más frágiles y muchas veces, al poseer una preservación más pobre, difícil de recuperar. Por otro lado, al incluir a todos los individuos mayores de 20 años en un solo grupo, se evitarían los posibles cuestionamientos respecto a la inexactitud de los métodos de estimación de la edad en adultos (Arias Fernández 2013; Bocquet-Appel y Masset 1977).

\section{Resultados}

El número mínimo de individuos estimados mediante la técnica de correspondencia (Todd y Frison 1992) para los entierros que aquí se consideran fue de $75(\mathrm{~N}=75)$. Es necesario aclarar que para la correspondencia anatómica individual no se consideraron los huesos de manos ni de pies ni los elementos costales, excepto para los entierros $\mathrm{H} 7 \mathrm{y}$ $\mathrm{H} 4$, donde el número de individuos inhumados y las notas de campo posibilitaron la asignación de estos elementos.

Los resultados obtenidos a partir de la aplicación de los estimadores de edad y sexo se muestran en la Tabla 3 y en la Figura 2, en las cuales también se puede observar la proporción de individuos por categoría sexo-etaria. Se observa que hay una proporción levemente mayor de individuos masculinos, la cual se hace evidente en el rango etario que va de los 20 a los 35 años (15 individuos masculinos y 10 femeninos). Además, este rango de edad es el más representado, ya que incluye al $33,33 \%$ de los

Tabla 2. Descripción de los parámetros paleodemográficos utilizados, tomados de Bocquet-Appel y Masset (1977). Description of the paleodemographic parameters used, taken from Bocquet-Appel and Masset (1977).

\begin{tabular}{|c|c|}
\hline Parámetro paleodemográfico & Cálculo según Bocquet-Appel y Masset (1977) \\
\hline Índice de juventud & $\mathrm{IJ}=\mathrm{D}_{5-14} / \mathrm{D}_{20-\infty}$ \\
\hline $\begin{array}{l}\text { Mortalidad promedio en los individuos juveniles (Mean } \\
\text { Childhood Mortality) MCM }\end{array}$ & $\mathrm{MCM}=(\mathrm{qx} 5-9,9+\mathrm{qx} 10-14,9+\mathrm{qx} 15-19,9) / 3$ \\
\hline Esperanza de vida al nacimiento $\mathrm{e}^{0}$ & $e^{0}=78,721 \times \log _{10} \sqrt{ }(1 / \mathrm{IJ})-3,384 \pm 1,503$ \\
\hline $\begin{array}{l}\text { Cociente de mortalidad infantil para el primer año de vida } \\
\text { (parámetro análogo a la tasa de mortalidad infantil (Jordana } \\
\text { Comin 2007)) }\end{array}$ & ${ }_{1} q_{0}=0,568 \times \sqrt{ } \log _{10}(200 \times I J)-0,438 \pm 0,016$ \\
\hline Cociente de mortalidad para el grupo de cero a cinco años & ${ }_{5} \mathrm{q}_{0}=1,154 \times \sqrt{ } \log _{10}(200 \times \mathrm{IJ})-1,014 \pm 0,041$ \\
\hline $\begin{array}{l}\text { Tasa anual de natalidad para poblaciones en crecimiento } \\
\text { moderado }\end{array}$ & $\mathrm{n}=0,166 \times \mathrm{IJ}+0,018 \pm 0,003$ \\
\hline $\begin{array}{l}\text { Tasa anual de mortalidad para poblaciones en crecimiento } \\
\text { moderado }\end{array}$ & $\mathrm{m}=0,117 \times \mathrm{IJ}+0,013 \pm 0,003$ \\
\hline $\begin{array}{l}\text { Tasa anual de natalidad y mortalidad en poblaciones para el } \\
\text { supuesto de poblaciones estacionarias }\end{array}$ & $\mathrm{n}=\mathrm{m}: \mathrm{n}=\mathrm{m}=0,127 \times \mathrm{IJ}+0,016 \pm 0,002$ \\
\hline $\begin{array}{l}\text { Tasa anual de fecundidad (población femenina en edad } \\
\text { reproductiva, es decir, entre } 15 \text { y } 50 \text { años) }\end{array}$ & ${ }_{35} \mathrm{~F}_{15}=-0,6601 \times \mathrm{IJ}^{0,5}+1,2419 \times \mathrm{IJ}^{0,3}-0,308 \pm 0,005^{*}$ \\
\hline $\begin{array}{l}\text { Índice de fertilidad (número de hijos por mujer, calculado } \\
\text { a partir de la tasa anual de fecundidad multiplicada por } 35 \\
\text { (duración estimada del periodo fértil de la mujer) }\end{array}$ & $\mathrm{IF}={ }_{35} \mathrm{~F}_{15^{*}} 35$ \\
\hline
\end{tabular}


Tabla 3. Proporción de individuos femeninos y masculinos adultos.

Proportion of adult female and male individuals.

\begin{tabular}{ccccccccccc}
\hline & $0-0,9$ & $1-4,9$ & $5-9,9$ & $10-14,9$ & $15-19,9$ & $20-34,9$ & $35-49,9$ & +50 & Total & \\
\hline $\mathrm{F}$ & 3 & 1 & - & 2 & 1 & 10 & 5 & 1 & 23 & $30,67 \%$ \\
$\mathrm{M}$ & 2 & 1 & 2 & 2 & 4 & 15 & 4 & - & 30 & $40 \%$ \\
$\mathrm{I}$ & 11 & 6 & 3 & 1 & 1 & - & - & - & 22 & $29,33 \%$ \\
\hline Total & 16 & 8 & 5 & 5 & 6 & 25 & 9 & 1 & 75 & $100 \%$ \\
\hline & $21,33 \%$ & $10,67 \%$ & $6,67 \%$ & $6,67 \%$ & $8 \%$ & $33,33 \%$ & $12 \%$ & $1,33 \%$ & $100 \%$ & \\
\hline
\end{tabular}

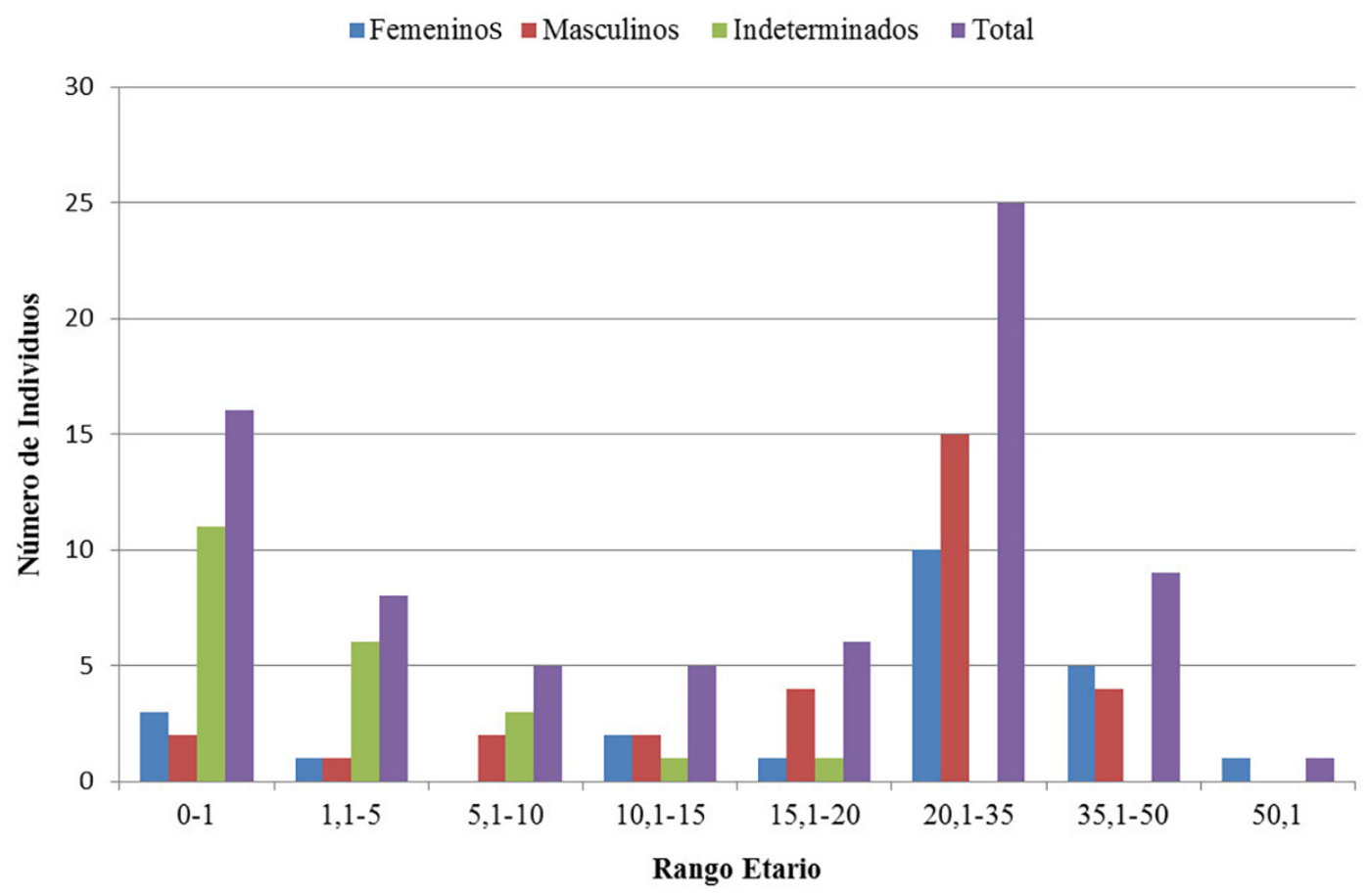

Figura 2. Distribución de individuos por categoría de edad.

Distribution of individuals by age category.

individuos de la muestra. El grupo que incluye a los individuos de 0-0,9 años de edad le sigue en cantidad de individuos. Dentro de este grupo, el 75\% ( $\mathrm{n}=12)$ corresponde a individuos cuyo deceso se produjo en el periodo próximo al nacimiento, poco tiempo antes, después o durante el mismo. Por lo tanto, el 16\% de todos los restos óseos analizados correspondería a perinatos. Finalmente, puede observarse que en esta muestra están representadas todas las categorías de edad y solo un individuo habría superado los 50 años.

A partir del número de individuos por rango etario se confeccionó la tabla de vida (Tabla 4). Mediante los valores de $1_{x}, \mathrm{q}_{\mathrm{x}} \mathrm{y} \mathrm{e}_{\mathrm{x}}^{\mathrm{o}}$ se elaboró la Figura 3, en la cual podemos ver las curvas de supervivencia, de probabilidad de fallecimiento y de esperanza de vida.

Siguiendo con el cálculo de indicadores paleodemográficos, el valor de la tasa bruta de mortalidad (M) es igual a 54,88 y el valor de la Tasa de Crecimiento Poblacional calculada fue de 1,5 por mil.

Por último, los resultados de los estimadores paleodemográficos propuestos por Bocquet-Appel y Masset (1977), calculados a partir del IJ, se exponen en la Tabla 5. El valor de IJ calculado para la muestra fue de 0,29 y el valor de MCM fue de 0,117. El número de hijos estimado por mujer que completó su ciclo reproductivo (IF) fue de 6,75 hijos. 
Tabla 4. Tabla de vida elaborada para el sitio Médano Petroquímica.

\begin{tabular}{|c|c|c|c|c|c|c|c|}
\hline Intervalo de edad & $\mathrm{D}_{\mathrm{x}}{ }^{2}$ & $\mathrm{~d}_{\mathrm{x}}{ }^{2}$ & $1_{\mathrm{x}}{ }^{2}$ & $\mathrm{q}_{\mathrm{x}}{ }^{2}$ & $\mathrm{~L}_{\mathrm{x}}{ }^{2}$ & $\mathrm{~T}_{\mathrm{x}}{ }^{2}$ & $\mathrm{e}_{\mathrm{x}}^{02}$ \\
\hline Perinato- 0,9 & 16 & 21,33 & 100 & 0,21 & 89,34 & 1822,46 & 18,22 \\
\hline $1-4,9$ & 8 & 10,67 & 78,67 & 0,14 & 293,34 & 1733,12 & 22 \\
\hline $5-9,9$ & 5 & 6,67 & 68 & 0,09 & 323,33 & 1439,78 & 21,17 \\
\hline $10-14,5$ & 5 & 6,67 & 61,33 & 0,11 & 289,98 & 1116,45 & 18,2 \\
\hline $15-19,5$ & 6 & 8 & 54,66 & 0,15 & 253,3 & 826,48 & 15,12 \\
\hline $20-34,9$ & 25 & 33,33 & 46,66 & 0,71 & 449,93 & 573,18 & 12,3 \\
\hline $35-49,9$ & 9 & 12 & 13,33 & 0,90 & 109,95 & 123,25 & 9,27 \\
\hline$>50$ & 1 & 1,33 & 1,33 & 1 & 13,3 & 13,3 & 10 \\
\hline
\end{tabular}

${ }^{2} \mathrm{Dx}: \mathrm{N}^{\circ}$ dedefunciones; $\mathrm{dx}$ \% \%dedefunciones;lx:supervivientes; qx:probabilidaddefallecimiento; Lx:años totales vividos entre el intervalo $\mathrm{x}$ y el siguiente; Tx: años totales restantes de vida; $\mathrm{e}_{\mathrm{x}}^{0}$ : esperanza de vida.

\section{- Esperanza de Vida - Supervivencia — Probabilidad de fallecimiento}

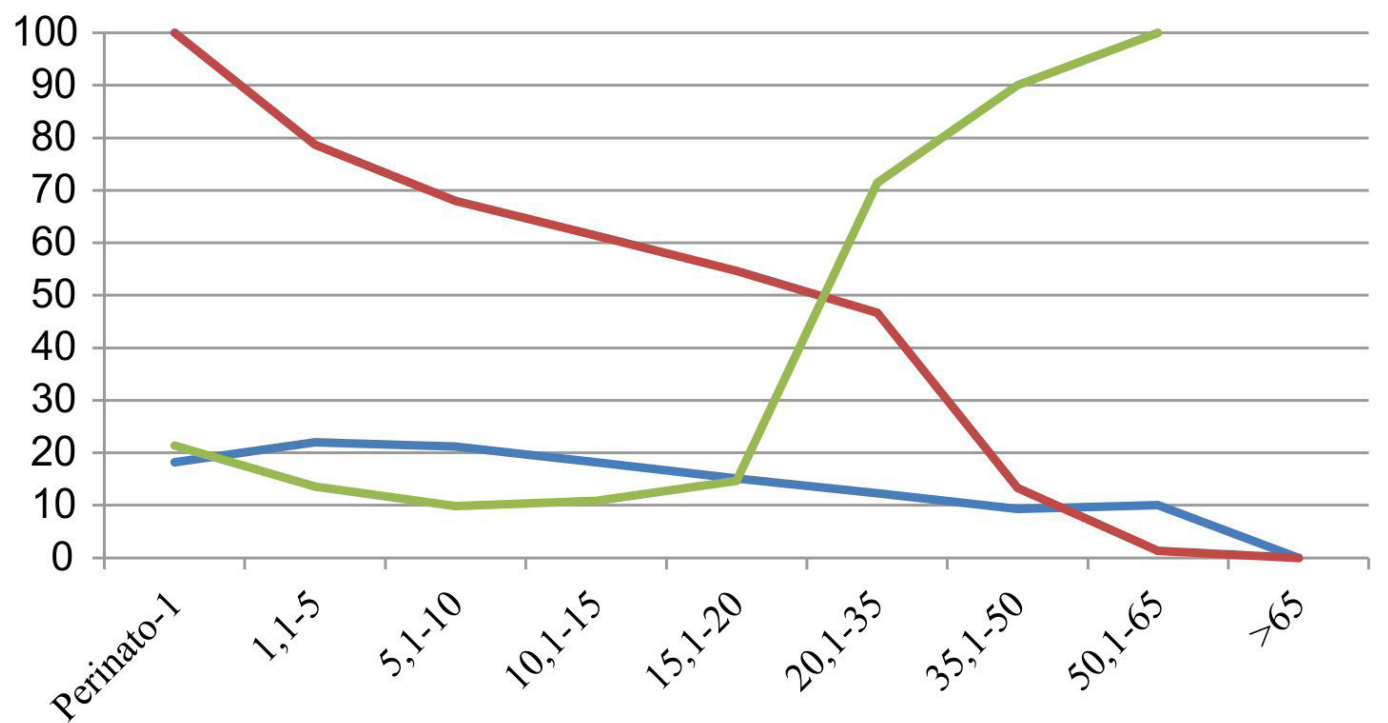

Figura 3. Curvas de supervivencia, de probabilidad de fallecimiento y de esperanza de vida construida a partir de los valores calculados de $1 \mathrm{x}$, qx y $\mathrm{e}_{\mathrm{x}}^{0}$, respectivamente.

Survival, probability of death and life-expectancy curves constructed from the calculated values of $l x$, qx, and $e^{o}{ }_{x}$, respectively.

Tabla 5. Estimadores paleodemográficos calculados para el sitio Médano Petroquímica. Paleodemographic estimators calculated for the Médano Petroquímica site.

\begin{tabular}{|c|c|c|c|c|c|c|c|c|c|}
\hline & $\mathrm{IJ}^{3}$ & MCM & $e^{03}$ & ${ }_{1} q_{0}{ }^{3}$ & ${ }_{5} \mathrm{q}_{0}{ }^{3}$ & $\mathrm{~m}=\mathrm{n}^{3}$ & $\mathrm{n}$ & $\mathrm{m}$ & ${ }_{35} \mathrm{~F}_{15}{ }^{3}$ \\
\hline Media & 0,29 & 0,117 & 17,776 & 0,316 & 0,518 & 0,053 & 0,066 & 0,046 & 0,193 \\
\hline Rango & & & $\begin{array}{l}16,273- \\
19,279\end{array}$ & $0,3-0,332$ & $0,477-0,559$ & $0,051-0,055$ & $0,063-0,069$ & $0,043-$ & $90,188-0,198$ \\
\hline
\end{tabular}

${ }_{3}^{3} \mathrm{IJ}$ : Índice de Juventud; $\mathrm{e}^{0}$ : Esperanza de vida al nacimiento; ${ }_{1} \mathrm{q}_{0}$ : cociente de mortalidad infantil para el primer año; ${ }_{5} \mathrm{q}_{0}$ : cociente de mortalidad para el grupo de 0 a 5 años; $\mathrm{n}$ : tasa anual de natalidad; $\mathrm{m}$ : tasa anual de mortalidad $\mathrm{y}_{35} \mathrm{~F}_{15}$ : tasa anual de fecundidad para las mujeres en edad reproductiva. 


\section{Discusión}

Además de la información que podemos extraer a partir de la construcción de perfiles en los que se muestra la distribución de edad y sexo de los individuos analizados, la elaboración de las tablas de vida es de gran utilidad, ya que estas permiten analizar a las poblaciones antiguas, particularmente en lo que respecta al costo adaptativo, a los factores que pudieron estar influyendo en la supervivencia de los distintos miembros del grupo, y, en definitiva, la calidad de vida del grupo analizado (Cocilovo et al. 1999).

En concordancia con estudios anteriormente realizados acerca de la composición etaria y sexual del sitio Médano Petroquímica, se observa que la distribución etaria se correspondería con un perfil de tipo atricional (Bernardi et al. 2016), presentando una distribución bimodal con picos para los infantes menores de un año y adultos jóvenes (Figura 2).

Antes de pasar al análisis de las tablas de vida, es necesario recordar los supuestos sobre los que se basa este tipo de estudios. En primer lugar, el uso de tablas de vida implica considerar que las poblaciones que originaron el sitio eran estacionarias. Al suponer que una población es estacionaria hacemos referencia a un grupo cerrado a la migración y donde la fecundidad y mortalidad se mantienen constantes (Wood et al. 1992). Este supuesto nos permite estandarizar los eventos demográficos de la población en estudio sin tener que recurrir a simulaciones de eventos como la migración (Hernández Espinoza 1999). Dado que la utilización de este supuesto nos lleva a una imagen no tan certera de las poblaciones prehistóricas, las cuales estaban abiertas a la migración, Roca (2013) discute la utilidad del concepto de estabilidad poblacional, denominando población estable a aquella en las cuales las tasas de fecundidad y mortalidad son invariantes por largos períodos de tiempo, manteniendo un ritmo de crecimiento constante. Otro de los supuestos a tener en cuenta es el uniformitarismo biológico (Howell 1976). Este concepto, tomado de las ciencias geológicas, hace referencia a que las respuestas biológicas del ser humano hacia el ambiente, tanto en el pasado como en la actualidad, bajo circunstancias similares, serían las mismas. Esto se aplica a ciertos parámetros demográficos como la mortalidad y fecundidad, donde no se supone que se hayan mantenido constantes a lo largo del tiempo, sino que responden de la misma manera a los cambios del medio (Howell 1976). Un tercer supuesto, relacionado a este último, hace referencia a la estimación de la edad a la muerte. $\mathrm{Si}$ bien las limitaciones que tienen las técnicas de estimación de edad han sido ampliamente reconocidas (por ej., mimetismo con la muestra de referencia, imprecisión en adultos, variaciones poblacionales, entre otras), algunos autores proponen utilizar el mayor número de técnicas disponibles para cada indicador y realizar una seriación en la cual la estimación de la edad reflejaría la posición etaria de un individuo en la muestra estudiada (Lovejoy et al. 1985; Storey 2007). Todos estos supuestos son necesarios para poder validar las inferencias que se realizan a partir de la muestra estudiada.

A partir del análisis de las tablas de vida elaboradas para Médano Petroquímica se puede observar que la curva de supervivientes, presentada en los resultados (Figura 3), decrece de manera continua y progresiva, presentando dos pendientes marcadas, una a partir del nacimiento y otra, más pronunciada aún, a partir de la categoría adulto joven. Esto coincide con la probabilidad de fallecimiento (qx), la cual aumenta notablemente a partir de los 20 años de edad. Respecto a la probabilidad de muerte, debemos destacar que, si bien el valor de qx aumenta con la edad, los individuos del rango etario que incluye desde momentos cercanos al nacimiento hasta el primer año de vida tienen mayor probabilidad de morir en comparación con el resto de los grupos de inmaduros. A su vez, los individuos de entre 1 y 4,9 años tienen un valor de qx mayor que el grupo siguiente (5-9,9 años). Es decir, y tal como lo muestra la Figura 3, vemos que una vez superado el año de edad, la probabilidad de morir para los individuos inmaduros empieza a disminuir hasta el período correspondiente a la adolescencia (rango de 10-14,9 años), a partir del cual nuevamente empieza a subir el riesgo de muerte, el mismo se eleva drásticamente a partir del comienzo de la edad adulta (20 años).

Con respecto a la esperanza de vida, esta fue calculada tanto mediante la utilización de los parámetros propios de la tabla de vida (Ubelaker 2007) como con el cálculo de los estimadores paleodemográficos propuestos a partir del IJ (Bocquet-Appel y Masset 1977). A partir de los datos de la tabla de vida podemos ver que la esperanza de vida al momento del nacimiento es de alrededor de 18 años y la probabilidad de muerte, de 0,21 (Figura 2). Los niños que superan el año de edad tienen una esperanza de vida de 22 años, es decir, una vez que se supera este umbral crítico, el cual se caracteriza por la inmadurez de los sistemas inmune y digestivo (Stinson 2000) y la necesidad 
de mayores cuidados por parte de los adultos, la esperanza de vida aumenta y, como mencionamos anteriormente, la probabilidad de muerte disminuye. El valor de la esperanza de vida al nacimiento calculado a partir de la propuesta de Bocquet-Appel y Masset (1977) fue de 17,77 años (con un rango de variación entre 16,27 y 19,28 años). Este valor se asemeja al calculado mediante la tabla de vida. Como el cálculo de la esperanza de vida basado en el IJ no tiene en cuenta al primer grupo de edad (para evitar sesgos por mala conservación o subrepresentación) y el estimado a partir de la tabla de vida sí incluye a este grupo, el haber obtenido valores de esperanza de vida al nacimiento tan similares a partir de ambas fórmulas nos estaría sugiriendo la existencia de una buena representación de inmaduros menores a un año de edad en el conjunto muestral, principalmente basada en una buena preservación y recuperación de sus elementos óseos (Iglesias Bexiga 2010). Estos valores concuerdan con los observados por Gage (2000) en varias poblaciones prehistóricas, en las cuales la esperanza de vida al nacer varía entre 18 y 25 años. Para el caso del cociente de mortalidad infantil (qx), calculado a partir de ambas metodologías, este fue similar para el primer año de vida $(0,21$ calculado mediante la tabla de vida y 0,31 sobre la base del IJ), pero bastante disímil para el rango comprendido entre el año y los cinco $(0,13$ calculado a partir de la tabla de vida y 0,51 mediante el IJ, que descarta este rango etario). Esta diferencia podría estar indicando una subrepresentación de individuos de entre 1 y 4,9 años. Gordón et al. (2019) obtuvieron valores similares de qx en el sitio Aquihuecó, localizado en la Provincia de Neuquén, aun cuando los rangos etarios adultos tenían una extensión distinta a la utilizada en este trabajo. La baja probabilidad de fallecimiento en los rangos etarios que abarcan los periodos de la niñez y la adolescencia podría estar indicando que los individuos de estas edades no estarían desarrollando actividades que los expusieran a mayores riesgos de morir. Esto es esperable ya que, en estas etapas, por lo general, se llevan a cabo fundamentalmente actividades de aprendizaje que les permitirán cumplir satisfactoriamente con sus tareas durante la vida adulta (Luna 2008). Sin embargo, el grupo que comprende el rango de 15-19,9 años seguramente ya desarrollaba la mayoría de las actividades propias de adultos. Por tal motivo, no debemos dejar de mencionar la respuesta diferencial a los estresores ambientales que presentan estos grupos etarios, la cual se traduce en una menor mortalidad (Bogin y Smith 2000).
Con respecto a la tasa bruta de mortalidad, el valor de la misma fue de 54,88 , lo cual nos estaría indicando que en la muestra bajo estudio murieron alrededor de 55 personas por cada mil cada año. Estos valores, al compararse con el de otras poblaciones, podrían revelar la existencia o no de diferencias y asî plantear nuevos problemas a investigar a nivel local y regional (Ubelaker 2007). Sin embargo, actualmente no estaríamos contando con estudios de otros sitios de la región en los cuales se haya estimado este parámetro.

La tasa anual de fecundidad para mujeres en edad reproductiva arrojó un valor promedio de 0,19 . Multiplicando este valor por 35 (cantidad de años promedio que duraría la vida reproductiva de la mujer), obtenemos el Índice de Fertilidad Femenina propuesto por Bocquet-Appel (1979). El mismo nos dio un valor de 6,75 , que equivaldría al número de nacimientos por mujer que sobrevive a todo su periodo reproductivo. Este valor es coherente con los reportados para otras poblaciones cazadorasrecolectoras (Lanza y Valeggia 2012). Un elevado valor del índice de fertilidad femenina, junto con una elevada mortalidad infantil, sugiere que se trata de una población con una elevada tasa de fecundidad (Konigsberg y Frakenberg 2002). En relación a esto, Wood et al. (1992), basándose en los trabajos de Sattenspiel y Harpending (1983) establecen que, en poblaciones no estacionarias, la esperanza de vida y la edad media de muerte son valores representativos de la fecundidad. En poblaciones estables (aquellas en las que los cambios en los valores de mortalidad y fecundidad se mantienen constantes), si los valores de esperanza de vida al nacimiento se mantienen constantes, cuando la edad media de muerte tenga valores bajos, la población presentará altos valores de fecundidad, y viceversa (Coale y Demeny 1983).

Siguiendo con los valores de fecundidad, Campbell y Wood (1988) llegaron a la conclusión de que las sociedades de pequeña escala, tales como las cazadoras-recolectoras, tenían un índice de fertilidad femenina promedio de seis (entre cuatro y ocho hijos). Esta información fue posteriormente validada por otros autores (Bentley et al. 1993a, 1993b; Pennington 2001). Respecto a esto, Kelly (1995) establece que el tiempo entre nacimientos es el factor más influyente en la fecundidad para este tipo de poblaciones. Los cazadores-recolectores necesitan tener intervalos largos entre nacimientos debido a la energía que insume el cuidado de niños durante la lactancia prolongada y la necesidad de cargarlos durante los procesos de movilidad grupal (Lee 1971; 
Sussman 1972). Si bien en una primera instancia se consideró la lactancia extendida como la principal causa del espaciamiento entre los nacimientos, el avance de las investigaciones permitió conocer que la lactancia suprime la ovulación solo durante los primeros 18 meses, alejándose del intervalo mínimo de 36 meses establecido para los cazadores-recolectores (Menken 1975). Este intervalo se explica, más allá de las razones de índole cultural, en la hipótesis de "grasa crítica" elaborada por Frisch $(1973,1976)$ y Frisch y McArthur (1974) y los desarrollos de Ellison (2003) sobre disponibilidad de energía materna, que establecen que la menarquia está asociada a un nivel determinado de grasa corporal y que, una vez alcanzada esta, el proceso de ovulación requiere un nivel de grasa mayor. Con cada nacimiento, si el niño sobrevive, la madre entra en un estado de requerimiento nutricional mayor debido a los costos de la lactancia, el cuidado y acarreo del niño y las actividades cotidianas de subsistencia. Según Howell (1976), una mujer puede necesitar hasta mil calorías diarias adicionales, que si no llegan a obtenerse harán que la mujer necesite más tiempo para alcanzar nuevamente el umbral de "grasa crítica" para el siguiente embarazo. Por otro lado, se estima que si el bebé no sobrevive, la mujeres podrían quedar embarazadas nuevamente en aproximadamente un año (Howell 1976).

En resumen, los trabajos de Frisch $(1973,1976)$ y Frisch y McArthur (1974) establecen que el nivel de fertilidad alcanzado por una determinada población depende directamente del medio en el que esta vive, además de los factores socioculturales que pueden estar influyendo y modelando este indicador paleodemográfico.

Por otro lado, Kelly (1995) propone un modelo que relaciona la disminución de la movilidad residencial y el aumento de la fecundidad. Esto concordaría con los cambios en la dinámica poblacional propuestos para las sociedades cazadoras-recolectores del área de estudio para el Holoceno Tardío (Berón y Politis 1997; Madrid y Barrientos 2000; Martínez 2006; Mazzanti y Quintana 2001) y con la aparición de áreas formales de entierro (Barrientos 2002). Si dejamos de lado por un momento el supuesto de estacionalidad, podemos calcular la tasa de crecimiento a partir de las fórmulas de Bocquet-Appel y Masset (1977). La tasa de crecimiento calculada para esta población es de 1,5 por mil, tal como se observa en otras poblaciones prehistóricas (Ramírez 2015). Esto es coherente con los resultados obtenidos a partir de la relación entre los valores de IJ y Mortalidad Promedio a la Niñez (Jackes 1992; Jackes 2011; Jackes et al. 1997). Los valores sugeridos por estos autores, por debajo de los cuales se puede evaluar el crecimiento de una población, son $\mathrm{IJ}=0,38$ (en nuestro caso de estudio el valor fue de 0,29 ) y $\mathrm{MCM}=0,135$ (siendo 0,117 para nuestro caso). Por otro lado, si calculamos las tasas de natalidad y mortalidad (según Bocquet-Appel y Masset 1977), suponiendo que no se trata de poblaciones estables, la tasa anual de natalidad $(0,066)$ es levemente mayor que la de mortalidad $(0,046)$. Por lo tanto, según estos cálculos, Médano Petroquímica se correspondería con una población en crecimiento.

Siguiendo con estos cambios de dinámica poblacional, no podemos dejar de mencionar la presencia de contingentes del Viejo Mundo en el continente americano. Si bien para los momentos que sugieren los fechados radiocarbónicos (entre 1400 y 1600 AD) hay escasos (o ninguno) registros de la presencia europea en el sudoeste de la Provincia de La Pampa, diversos puntos del territorio argentino ya contaban con asentamientos españoles (la ciudad de Buenos Aires tuvo su primera fundación en el año 1536). El hallazgo de una cuenta de vidrio veneciana en la base de uno de los entierros sugiere que la sociedad bajo estudio ya conocía de la llegada de los colonizadores europeos a través de las redes de circulación de bienes propuestas para Norpatagonia, el centro-sur de Chile, Cuyo, la región pampeana y la costa atlántica. La presencia de los europeos muy probablemente habría sido otro importante factor influyente en la dinámica y movilidad poblacional de estas sociedades. La interacción de las sociedades pampeanas y europeas tuvo como consecuencias cambios en la organización de los nativos, modificaciones en la economía de subsistencia, incorporación de nuevas tecnologías, cambios en los patrones de movilidad y uso del espacio, conflictos armados, cambios demográficos en los patrones de morbilidad y mortalidad, entre otros (Oliva et al. 2007). Aunque no tenemos certeza de que la presencia de europeos haya ejercido una influencia directa, estimamos que las distintas presiones a las que fueron sometidas las sociedades más cercanas a los asentamientos europeos habrían generado un efecto en cascada que habría llegado incluso a afectar a las poblaciones más alejadas de estos asentamientos.

Finalmente, consideramos necesario destacar que el análisis efectuado en este trabajo tendría gran relevancia para el estudio de las estructuras y dinámicas poblacionales de las sociedades de cazadores-recolectores del Holoceno Tardío de las regiones Pampa y Patagonia de Argentina. Por un lado, para esta investigación se consideró un total de 75 individuos esqueletales, un número relativamente grande y muy poco común para sitios con entierros de cazadores-recolectores en estas regiones. Si bien la cantidad de sitios asociados a cazadores-recolectores en el sur de Argentina es elevada, la gran mayoría de 
ellos están representados por entierros aislados con un bajo número de inhumados, entre los cuales podemos mencionar los sitios del sur de la Patagonia (Suby et al. 2017), los entierros de la localidad arqueológica Barrancas (Novellino et al. 2013), el sitio Loma de la Lata (Cúneo et al. 2015), Cañada Seca (Peralta 2019) y el sitio La Petrona (Flengsborg et al. 2011). Como excepción podemos mencionar los sitios Chenque I, en el centro de la Provincia de La Pampa, con 164 individuos inhumados entre dos submuestras (Luna 2008); el sitio Paso Alsina en el sur de la Provincia de Buenos Aires, del cual se han recuperado 77 individuos (Flensborg 2012); los sitios de la cuenca del Lago Salitroso en la Provincia de Santa Cruz, con 71 esqueletos pertenecientes a los chenques tardíos (800-350 años AP) (García Guraieb 2010; García Guraieb et al. 2015), y el sitio Aquihuecó, en la Provincia de Neuquén, con un MNI de 64 (Gordón et al. 2019). Por otro lado, seis fechados radiocarbónicos efectuados sobre muestras de hueso procedentes de los distintos rasgos que conforman este sitio sugieren que el Médano Petroquímica habría sido utilizado durante un periodo acotado (entre los años 363 y 419 AP), lo cual no nos permite desestimar la posibilidad de que se trate de un único evento sincrónico de inhumación. De este modo, estas propiedades apuntan a que este sitio con entierros podría resultar, en cierto grado, representativo de la/s sociedad/es que lo generó, volviéndolo mayormente idóneo para llevar a cabo análisis paleodemográficos y realizar el cálculo de distintos parámetros poblacionales.

Sin embargo, más allá de que las características del sitio Médano Petroquímica apoyen la utilización de las tablas de vida, somos plenamente conscientes de que hemos considerado un conjunto de supuestos y que también se depende de la exactitud de los métodos de estimación de edad y sexo (Ubelaker 2007). Por lo tanto, las inferencias realizadas a partir de los resultados obtenidos no deben ser consideradas como evidencias indiscutibles acerca de esta/s sociedad/es, sino más bien como una propuesta de interpretaciones acerca de las distintas propiedades demográficas del conjunto en estudio.

\section{Conclusiones}

Este trabajo constituye uno de los primeros estudios en incluir la utilización de las tablas de vida para el análisis paleodemográfico en el área. A partir de los resultados obtenidos se puede observar la existencia de una buena representación de las categorías etarias consideradas. La distribución etaria se correspondería con un perfil de tipo atricional, semejante al de otras poblaciones cazadorasrecolectoras de esta y otras regiones, con picos en los rangos correspondientes a individuos menores a un año y adultos jóvenes. La esperanza de vida al nacer calculada ronda los 18 años y aumenta luego de superado el periodo crítico del primer año de vida, cuando la probabilidad de fallecimiento es mayor probablemente debido a la inmadurez del sistema inmune y la alta dependencia del cuidado adulto por parte de los infantes. Los individuos correspondientes al rango de 20-34,9 años también tienen una elevada probabilidad de fallecimiento, pero en este caso este valor podría estar respondiendo al desarrollo de actividades de mantención y defensa del grupo y, en el caso de las mujeres, reproducción.

Por otro lado, los distintos indicadores paleodemográficos utilizados (tasa anual de fecundidad, índice de fertilidad, relación entre el índice de juventud y la mortalidad promedio a la niñez, tasa de natalidad y mortalidad) estarían indicando que durante este periodo la población habría estado en crecimiento, lo cual es coincidente con las propuestas de reducción de movilidad y aumento de la densidad poblacional para la región durante el Holoceno Tardío.

Una vez más cabe destacar la importancia del empleo de estas herramientas (tablas de vida y otros parámetros paleodemográficos) en los estudios paleodemográficos, los cuales representan una línea de análisis de suma relevancia para hacer inferencias de las poblaciones del pasado, más allá de las severas críticas a las cuales han estado y están sometidos. Además, estas inferencias pueden resultar de gran importancia cuando se trata de muestras esqueléticas que tienen un número elevado de individuos, una buena representación de todos los grupos etarios y proceden de contextos que sugieren un uso acotado en el tiempo del sitio de entierro, tal como sería el caso en el Médano Petroquímica.

Agradecimientos: Los autores agradecen a la Secretaría de Cultura de la Provincia de La Pampa y al CONICET. A la Dra. Ana Margarita Aguerre y al Lic. Lía Pera. Además, agradecemos a los revisores anónimos por las sugerencias realizadas que contribuyeron a mejorar enormemente este documento.

\section{Referencias Citadas}

Acsádi, G. y J. Nemeskéri 1970. History of Human Life Span and Mortality. Akadémiai Kiadó, Budapest.
Angel, J.L. 1969. The bases of paleodemography. American Journal of Physical Anthropology 30:427-438. 
Arias Fernández, M. 2013. Estudio de la Juventud en la España Peninsular e Islas Baleares desde el Imperio Romano a la Edad Moderna. La Demografía llega a la Paleodemografía. Tesis de Maestría, Máster conjunto entre la Universidad de Alcalá, Autónoma de Madrid y Complutense de Madrid, Madrid.

Barrientos, G. 2001. Una aproximación bioarqueológica al estudio del poblamiento prehispánico tardío del sudeste de la región pampeana. Intersecciones en Antropología 2:3-18.

Barrientos G. 2002. The archaeological analysis of death-related behaviors from an evolutionary perspective: exploring the bioarchaeological record of early American hunter-gatherers. En Perspectivas Integradoras entre Arqueología y Evolución. Teoría, Métodos y Casos de Aplicación, editado por G. Martínez y L. Lanata, pp. 221-253. INCUAPA, Universidad Nacional del Centro, Olavarría.

Barrientos, G. e I. Pérez 2004. La expansión y la dispersión de poblaciones del norte de Patagonia durante el holoceno tardío: evidencia arqueológica y modelo explicativo. En Contra Viento y Marea. Arqueología de la Patagonia, editado por M.T. Civalero, P.M. Fernández y A.G. Guraieb, pp. 179-195, INAPL y SAA, Buenos Aires.

Begon, M., J.L. Harper y C.R. Townsend 1995. Ecología: Individuos, Poblaciones y Comunidades. Ediciones Omega, Barcelona.

Bentley, G.R., T, Goldberg y G. Jasieñska 1993a. The fertility of agricultural and nonagricultural traditional societies. Population Studies 47:269-281.

Bentley, G.R, G. Jasieñska y T. Goldberg 1993b. Is the fertility of agriculturalists higher than that of nonagriculturalists? Current Anthropology 34:778-785.

Bernal, V., S. García Guraieb y P. González 2004. Perfiles de mortalidad de las muestras de restos óseos humanos procedentes del área del lago Salitroso (provincia de Santa Cruz). En Contra Viento y Marea, editado por M. T. Civalero, P. Fernández y A. G. Guraieb, pp. 361-373. Instituto Nacional de Antropología y Pensamiento Latinoamericano, Buenos Aires.

Bernardi, L. 2018. Los cazadores-recolectores del Médano Petroquímica (Colonia 25 de Mayo, Puelén, La Pampa). Caracterización de la Estructura y Composición del Conjunto por medio de Marcadores Anatomofuncionales. Tesis doctoral inédita, Facultad de Ciencias Exactas, Físico-Químicas y Naturales, Universidad Nacional de Río Cuarto, Río Cuarto.

Bernardi, L. 2019. Evaluación de estimadores de edad y sexo en coxales adultos del sitio Médano Petroquímica. En Metodologías para el Estudio de Restos Esqueletales en Argentina: Actualizaciones y Nuevas Perspectivas, editado por M. Fabra, P.S. Novellino, M.A. Arrieta y M.S. Salega, pp. 182-205. Facultad de Filosofía y Humanidades, Universidad Nacional de Córdoba.

Bernardi, L., M. Arrieta, M.C. Bottini y O.J. Mendonça 2016. El sitio Médano Petroquímica (Puelén, La Pampa). Una Aproximación Paleodemográfica. Actas del XCX Congreso Nacional de Arqueología Argentina, pp. 1308-1311. Facultad de Ciencias Naturales e I.M.L. Universidad Nacional de Tucumán, Tucumán.

Berón, M. y G. Politis 1997. Arqueología pampeana en la década de los 90', estado de las investigaciones y perspectivas. En Arqueología Pampeana en la Década de los 90', editado por M. Berón y G. Politis, pp. 7-32. INCUAPA, Facultad de Ciencias
Sociales de Olavarría, Universidad Nacional del Centro. Museo de Historia Natural de San Rafael, Olavarría y San Rafael.

Bocquet-Appel, J.P. 1979. Une approche de la fécondité des populations inhumées. Bulletins et Mémoires de la Sociétéd'Anthropologie de Paris 6:261-268.

Bocquet-Appel, J.P. y C. Masset 1977. Estimateur en paléodémographie. L'Homme 27:65-90.

Bottini M.C., M.A. Arrieta y L. Bernardi 2020. El comportamiento mortuorio en los cazadores-recolectores del sitio Médano Petroquímica (La Pampa, Argentina). Caracterización preliminar desde un abordaje tafonómico. Chungara Revista de Antropología Chilena 52 (2):241-259.

Bogin, B. y B.H. Smith 2000. Evolution of the human life cycle. En Human Biology: An Evolutionary and Biocultural Perspective, editado por S. Stinson, B. Bogin, R. Huss-Ashmore y D. O'Rourke, pp. 377-424. Wiley-Liss, New York.

Brook, S. y J.M. Suchey 1990. Skeletal age determination base on the os pubis: A comparison of the Acsádi-Nemerkéri and Suchey-Brooks methods. Human Evolution 5 (3):227-238.

Buckberry, J. y A. Chamberlain 2002. Age estimation from the auricular surface of the ilium: A revised method. American Journal of Physical Anthropology 119:231-239.

Buikstra, J.E. y L.W. Konigsberg 1985. Paleodemography: Critiques and commentaries. American Anthropologist 87:316-333.

Buikstra, J. y J. Mielke 1985. Demography, diet and health. En The Analysis of Prehistoric Diets, editado por R. Gilbert y J. Mielke, pp. 360-422. Academic Press, Orlando.

Buikstra, J.E. y D.H. Ubelaker (eds.) 1994. Standard for Data Collection from Human Skeletal Remains. Arkansas Archeological Survey Research Series No 44. Fayetteville.

Campbell, K.L. y J.W. Wood. 1988. Fertility in traditional societies. En Natural Human Fertility: Social and Biological Mechanism, editado por P. Diggory, S. T'eper y M. Potts, pp. 39-69. Macmillan, London.

Chamberlain, A.T. 2006. Demography in archaeology. Cambridge University Press, Cambridge.

Coale, A.J. y J.P. Demeny 1983. Regional Model Life Tables and Stable Populations (second edition). Academic Press, Nueva York.

Cocilovo, J.A., S.G. Valdano y H.H. Varela 1999. Estimaciones de parámetros demográficos en poblaciones nativas antiguas y contemporáneas. Revista Argentina de Antropología Biológica 2 (1):27-40.

Cúneo, E.M., A. Hajduk, P.S. Novellino y P.F. Azar 2015. Rescate de un cementerio de cazadores-recolectores prehispánicos: sitio Loma de la Lata (provincia del Neuquén, República Argentina). Intersecciones en Antropología 17:315-325.

Ellison, P.T. 2003. Energetics and reproductive effort. American Journal of Human Biology 15 (3):342-351.

Fazekas, I.G. y F. Kósa 1978. Forensic Fetal Osteology. Akadémiai Kiadó, Budapest.

FitzgGerald, C., S. Saunders, L. Bondioli y R. Macchiarelli 2006. Health of infants in an imperial roman skeletal sample: perspective from dental microstructure. American Journal of Physical Anthropology 130:179-189. 
Flensborg, G. 2012. Análisis Paleopatológico en el Curso Inferior del Río Colorado (Provincia de Buenos Aires). Exploración y Evaluación del Estado de Salud de Sociedades CazadorasRecolectoras en el Holoceno Tardío. Tesis de doctorado en arqueología, Facultad de Ciencias Sociales, Universidad Nacional del Centro de la Provincia de Buenos Aires, Buenos Aires.

Flensborg, G. y P. Bayala 2017. Bioarqueología. Sexo, edad, salud y patrones de mortalidad. En Arqueología de CazadoresRecolectores del Curso Inferior del Río Colorado, editado por G. Martínez, pp. 146-174. INCUAPA, CONICET, Universidad Nacional del Centro, Olavarría.

Flensborg, G., G. Martínez y P. Bayala 2015. Mortality profiles of hunter-gatherer societies: a case study from the eastern PampaPatagonia transition (Argentina) during the final late Holocene. International Journal of Osteoarchaeology 25 (6):816-826.

Flensborg, G., G. Martínez, M. González y P. Bayala 2011. Revisión de los restos óseos humanos del sitio La Petrona (Transición Pampeano-Patagónica oriental, Argentina). Magallania 39 (1):179-191.

Frankenberg, S.R. y L.W. Konigsberg 2006. A brief history of paleodemography from Hooton to Hazards Analysis. En Bioarchaeology. The Contextual Analysis of Human Remains, editado por L.A. Beck y J.E. Buikstra, pp. 227-261. Elsevier Press, New York.

Frisch, R.E. 1973. The critical weight at menarche and the initiation of the adolescent growth spurt, and the control of puberty. En The Control of the Onset of Puberty, editado por M. Grumback, G. Grave y F. Mayer, pp. 403-423. Wiley-Interscience, New York.

Frisch, R.E. 1976. Demographic implications of the biological determinants of female fecundity. Social Biology 22 (1):17-22.

Frisch, R.E. y J.W. McArthur 1974. Menstrual cycle: fatness as a determinant of minimum weight for height necessary for their maintenance or onset. Science 185 (4155):949-951.

Gage, T.B. 2000. Demography. En Human Biology: An Evolutionary and Biocultural Perspective, editado por S. Stinson, B. Bogin, R Huss-Ashmore y D. O'Rourke, pp. 507-551. WileyLiss, New York.

García Guraieb, S. 2010. Bioarqueología de CazadoresRecolectores del Holoceno Tardío de la Cuenca del Lago Salitroso (Santa Cruz): Aspectos Paleodemográficos y Paleopatológicos. Tesis doctoral inédita, Facultad de Filosofía y Letras, Universidad de Buenos Aires, Buenos Aires.

García Guraieb, S., R. Goni y A. Tessone 2015. Paleodemography of Late Holocene hunter-gatherers from Patagonia (Santa Cruz, Argentina): An approach using multiple archaeological and bioarchaeological indicators. Quaternary International 356:147-158.

Gilbert, B. y T. McKern 1973. A method for aging the female os pubis. American Journal of Physical Anthropology 38:31-38.

Gordón, F., M. Béguelin, P. Novellino y F. Archuby 2019. Inferencias paleodemográficas en el Noroeste de Patagonia a partir del sitio Aquihuecó, Provincia del Neuquén, Argentina. Chungara Revista de Antropología Chilena 51 (3):363-380.

Hernández Espinoza, P.O. 1999. Los estudios paleodemográficos en México. Revista Argentina de Antropología Biológica 2 (1):335-355.
Hoppa, R.D. 2002. Paleodemography: Looking back and thinking ahead. En Paleodemography: Age Distributions from Skeletal Samples, editado por R.D. Hoppa y J.W. Vaupel, pp. 9-28. Cambridge University Press, Cambridge.

Howell, N. 1976. Toward uniformitarian theory of human paleodemography. Journal of Human Evolution 5:25-40.

Iglesias Bexiga, J. 2010. Primera Aproximación a las Características Demográficas del Cementerio Hispanomusulmán de San Nicolás. Ávila (S. XII-XVI). Tesis de Maestría, Universidad de Alcalá, Universidad Complutense de Madrid y Universidad Autónoma de Madrid, Madrid.

Jackes, M. 1986 The mortality of Ontario archaeological populations. Canadian Journal of Anthropology 5:33-48.

Jackes, M. 1992 Paleodemography: problems and techniques. En Skeletal Biology of Past Peoples: Research Methods, editado por S.R. Saunders y M.A. Katzenberg, pp. 189-224. Wiley-Liss, New York.

Jackes, M. 2011. Representativeness and bias in archaeological skeletal samples. En Social Bioarchaeology, editado por S. Agarwal y B. Glencross, pp. 107-146. Wiley-Blackwell, Oxford.

Jackes, M., D. Lubell y C. Meilejohn 1997. Healthy but moral: Humanbiology and the first farmers of Western Europe. American Antiquity 71:639-658.

Johnston, F.E. 1962. Growth of the long bones of infants and young children at Indian Knoll. American Journal of Physical Anthropology 20:249-254.

Kelly, R. 1995. The Foraging Spectrum: Diversity in HunterGatherer Lifeways. Smithsonian Institution Press, Washington DC.

Konigsberg, L.W. y S.R. Frankenberg 2002. Deconstructing death in paleodemography. American Journal of Physical Anthropology 117:297-309.

Krogman, W.M. y M.Y. Isçan 1986. The Human Skeleton in Forensic Medicine. Thomas Publisher, Illinois.

Lanza, N. y C. Valeggia 2012. La modernización ¿Siempre disminuye la fecundidad? Análisis de la transición demográfica en una población Toba. Revista Argentina de Antropología Biológica 14 (1):33-43.

Lee, M. 1971. Maturation disparity between hand-wrist bones in Hong Kong Chinese children. American Journal of Physical Anthropology 34:385-96.

Loth, S. y M. Henneberg 1996. Mandibular ramus flexure: a new morphologic indicator of sexual dimorphism in the human skeleton. American Journal of Physical Anthropology 99:473-485.

Loth, S.R. y M. Henneberg 2001. Sexually dimorphic mandibular morphology in the first few years of life. American Journal of Physical Anthropology 115 (2):179-186.

Lovejoy, C.O. 1985. Dental wear in the Libben population: Its functional pattern and role in the determination of adult skeletal age at death. American Journal of Physical Anthropology 68 (10):47-56.

Lovejoy, C., R. Meindl, R. Mensforth y T. Barton 1985. Multifactorial determination of skeletal age at death: A method and blind tests of its accuracy. American Journal of Physical Anthropology 68:1-14. 
Luna, L.H. 2008. Estructura Demográfica y Relaciones Biológicas de Cazadores Recolectores en un Ambiente de Desierto. Sitio Chenque I (Parque Nacional Lihué Calel, Provincia de La Pampa, Argentina). Tesis doctoral. BAR Internacional Series, Archaeopress, Oxford.

Luna, L.H. 2012. Validación de métodos para la generación de perfiles de mortalidad a través de la dentición. Su importancia para la caracterización paleodemográfica. Revista Argentina de Antropología Biológica 14:33-51.

Lynch Ianniello, I., M.A. Arrieta, L. Bernardi y O.J. Mendonça 2019. Paleodietary trends in Late Holocene Hunter-Gatherers from Southwestern La Pampa Province, Argentina. First results from the Médano Petroquímica site through Stable Isotope Analysis ( $\delta 13 \mathrm{C}$ and $\delta 15 \mathrm{~N})$. Quaternary International 548:84-94.

Madrid, P. y G. Barrientos 2000. La estructura del registro arqueológico Laguna Tres Reyes 1 (provincia de Buenos Aires): nuevos datos para la interpretación del poblamiento humano del sudeste de la Región Pampeana a inicios del Holoceno Tardío. Relaciones de la Sociedad Argentina de Antropología 25:179-206.

Martella, M.B., E.V. Trumper, L.M. Bellis, D. Renison, P.F. Giordano, G. Bazzano y R.M. Gleiser 2012. Manual de ecología poblaciones: demografía, crecimiento e interacciones. Reduca (Biología). Serie Ecología 5 (1):32-70.

Martínez, G. 2006. Arqueología del curso medio del río Quequén Grande: estado actual y aportes a la arqueología de la región pampeana. Relaciones de la Sociedad Argentina de Antropología 31:249-275.

Mays, S.A. 2002. Asymmetry in metacarpal cortical bone in a Collection of British Post-Medieval human skeleton. Journal of Archaeological Science 29:435-441.

Mazzanti, D. y C. Quintana 2001. Cueva Tixi: Cazadores y Recolectores de las Sierras de Tandilia Oriental. Geología, Paleontología y Zooarqueología. Laboratorio de Arqueología, Universidad Nacional de Mar del Plata, Mar del Plata.

McKern, T. y T. Stewart 1957. Skeletal age changes in young American males. Headquarters, Quartermaster Research \& Development Command, Natick.

Meindl, R. y C. Lovejoy 1989.Age changes in the pelvis: implications for paleodemography. En Age Markers in the Human Skeleton, editado por M. Isçan, pp. 137-168. Charles C. Thomas, Springfield.

Meindl, R. y K. Russell 1998. Recent advances in method and theory in paleodemography. Annual Review of Anthropology 27:375-399.

Menken, JA. 1975. Estimating Fecundability. Tesis doctoral inédita. Princeton University, New Jersey.

Mendonça, O.J., A.M. Aguerre, M.A. Bordach, M.G. Ammann, M.A. Arrieta, M.C. Croatto y L.M. Pera 2010. Inclusiones funerarias y dimensiones sociales del comportamiento mortuorio en el Médano Petroquímica, departamento. Puelén, provincia de La Pampa. En Mamül Mapu: Pasado y Presente desde la Arqueología Pampeana, editado por M. Berón, L. Luna, M. Bonomo, C. Montalvo, C. Aranda y M. Carrera Aizpitarte, T.1, pp. 223-230. Libros del Espinillo, Ayacucho.

Milner, G.R., D.A. Humpf y H.C. Harpending 1989. Pattern matching of age-at-death distributions in paleodemographic analysis. American Journal of Physical Anthropology 80:49-58.
Milner, G.R.1992. Determination of skeletal age and sex: a manual prepared for the Dickson Mounds Reburial Team. Ms. of file. Dickson Mounds Museum, Lewistown Ill.

Molleson, T., K. Cruse y S. Mays 1998. Some sexually dimorphic features of the human juvenile skull and their value in sex determination in inmature skeletal remains. Journal of Archaeological Science 25 (8):719-728.

Moore, J.A., A.C. Swedlund y G.J. Armelagos 1975. The use of life tables in paleodemography. American Antiquity 40 (2):57-70.

Novellino, P.S., N.B. Aporta, L.A. D'Addona, D. Estrella, V. Bernal, S. Devincenzi y V. Durán 2013. Sitios de entierro de la localidad arqueológica Barrancas, Maipú (Mendoza, Argentina). Intersecciones en Antropología 14 (1):271-277.

Oliva, F., G. L'Heureux, H. De Ángelis, V. Parmigiani y F. Reyes 2007. Poblaciones indígenas de momentos postcontacto en el borde occidental de la pampa húmeda: Gascón 1, un sitio de entierros humanos. Arqueología Argentina en los Inicios de un Nuevo Siglo 1:265-274.

Osterholtz, A.J. 2019. Advances in documentation of commingled and fragmentary remains. Advances in Archaeological Practice 7 (1):77-86.

Pennington, R. 2001. Hunter-gatherer demography. En HunterGatherers: An Interdisciplinary Perspective, editado por C. Panter-Brick, R.H. Layton y P. Rowley-Conwy, pp. 170-204. Cambridge University Press, Cambridge.

Peralta, E.A. 2019. Demografía Humana, Dieta y Actividad en los Límites de la Dispersión Agrícola: Tendencias Bioarqueológicas en el Sur de Mendoza a Finales de Holoceno Tardio. Tesis doctoral en Arqueología, Facultad de Filosofía y Letras, Universidad de Buenos Aires, Buenos Aires.

Phenice, T.W. 1969. A newly development visual method of sexing the os pubis. American Journal of Physical Anthropology 30:297-301.

Ramírez, J.P.D. 2015. Estudio Bioantropológico Comparado de Tres Necrópolis Históricas Excavadas en el Término Municipal de Lucena (Córdoba). Tesis doctoral, Facultad de Medicina, Universidad de Granada, Granada.

Roca, M.G. 2013. Paleodemografía: problemas metodológicos y perspectivas. Cuadernos de Prehistoria y Arqueología de la Universidad de Granada 22:67-77.

Rodríguez Cuenca, J.V. 1994. Introducción a la Antropología Forense. Análisis e Identificación de Restos Óseos Humanos. Departamento de Antropología, Universidad Nacional de Colombia, Bogotá.

Sánchez Romero, M. 2006. Maternidad y Prehistoria: prácticas de reproducción, relación y socialización. En Las Mujeres en la Prehistoria, coordinado por Soler Mayor, pp. 119-138. Museu de Prehistòria de València, Valencia.

Sattenspiel, L. y H.C. Harpending 1983. Stable population and skeletal age. American Antiquity 48:489-498.

Scheuer, L. y S. Black 2000. Developmental Juvenile Osteology. Academic Press, Londres.

Schutkowski, H. 1993. Sex determination of infant and juvenile skeletons: I. Morphognostic features. American Journal of Physical Anthropology 90:199-205. 
Stinson, S. 2000. Growth variation: Biological and cultural factors. En Human Biology: An Evolutionary and Biocultural Perspective, editado por S. Stinson, P. Bogin, Huss-Ashmore y D. O'Rourke, pp. 425-463. Wiley-Liss, New York.

Storey, R. 2007. An elusive paleodemography? A comparison of two methods for estimating the adult age distribution of deaths at late Classic Copan, Honduras. American Journal of Physical Anthropology 132:40-47.

Suby, J.A., L.H. Luna, C.M. Aranda y G.A. Flensborg 2017. First approximation to paleodemography through age-at-death profiles in hunter-gatherers from Southern Patagonia during middle-late Holocene. Quaternary International 438:174-188.

Sussman, R.W. 1972. Child transport, family size and increase in human population during the Neolithic. Current Anthropology 13 (2):258-259.

Sutherland, L. y J. Suchey 1991.Use of the ventral arc in pubic sex determination. Journal of Forensic Sciences 36 (2):501-511.

Todd, T. 1921a. Age changes in the pubic bone. I: the male white pubis. American Journal of Physical Anthropology 3:285-334.
Todd, T. 1921b. Age changes in the pubic bone. III: the pubis of the white female. IV: the pubis of the female white-negro hybrid. American Journal of Physical Anthropology 4:1-70.

Todd, L. y G. Frison 1992. Reassembly of bison skeletons from the Horner Site: A study in anatomical refitting. En Piecing Together the Past: Applications of Refitting Studies in Archaeology, editado por J. Hofman y J. Enloe, pp. 63-82. British Archaeological Reports, Oxford.

Ubelaker, D.H. 1999. Human Skeletal Remains; Excavation Analysis, Interpretation. 3rd edition. Taraxacum, Washington DC.

Ubelaker, D.H. 2007. Enterramientos Humanos. Excavación, Análisis, Interpretación. Sociedad de Ciencias Aranzadi, Donostia.

Volk, A.A. y J.A. Atkinson 2013. Infant and child death in the human environment of evolutionary adaptation. Evolution and Human Behavior 34 (3):182-192.

Walker, P. 2005. Greater sciatic notch morphology: Sex, age, andn population differences. American Journal of Physical Anthropology 127:385-391

Wood, J.W., G.R. Milner, H.C. Harpending y K.M. Weiss 1992. The Osteological Paradox: problems of inferring prehistoric health from skeletal samples. Current Anthropology 33 (4):343-370. 
